\title{
Radiology-Pathology Correlation of Rectal Cancer with Magnetic Resonance (MR) Imaging and Whole-mount Pathologic Specimen; General Review of Rectal MR Imaging and Clinical Implications
}

Seong Jin Park, Sung Kyoung Moon, Dong Ho Lee, Joo Won Lim Department of Radiology, Kyung Hee University Hospital, Seoul, Korea

The major purpose of this educational exhibition is to demonstrate the educational cases with rectal MR imaging and whole-mount specimen for radiology-pathology correlation.

Introduction

An accurate staging and description of tumor location is important for treatment planning. Early stage rectal cancer (cT1 \& cT2) and some advanced rectal cancer can be cured by local excision (TME or ESD) by resection with negative margins. Positive surgical margin is closely related with local tumor recurrence and reduced disease-free survival. Advanced rectal cancer with deep cancer invasion, lower rectal cancer neighboring or involving the anal sphincter, lymph node metastasis, or distant metastasis disease should be treated by other treatment options such as preoperative chemoradiation therapy (CRT), systemic chemotherapy, or local radiation therapy. Rectal MRI plays a critical role in posttreatment evaluation for the treatment of choice by the assessment of clinical restaging, treatment response, remaining cancer burdens, and surgical extent..

\begin{tabular}{|l|}
\hline Rectal MRI techniques \\
- ESGAR Guidelines - \\
\hline
\end{tabular}

\section{Hardware and patient preparation}

\begin{tabular}{|c|c|c|}
\hline \multicolumn{2}{|l|}{ Field strength } & 1.5 or $3.0 \mathrm{~T}$, minimum $1.0 \mathrm{~T}$ \\
\hline \multicolumn{2}{|l|}{ Coil } & Surface coil, not endorectal coil \\
\hline \multicolumn{2}{|c|}{ Endorectal filling or enema } & Not routinely recommended \\
\hline \multicolumn{3}{|l|}{ Sequences } \\
\hline Routine & \multicolumn{2}{|c|}{ 2D T2WI } \\
\hline DWI & \multicolumn{2}{|c|}{ Not obligatory for the initial staging } \\
\hline Others & \multicolumn{2}{|c|}{$\begin{array}{l}\text { 3D T2WI, fat suppression, contrast } \\
\text { enhancement, and steady state sequences are } \\
\text { not recommended. }\end{array}$} \\
\hline Planes & \multicolumn{2}{|c|}{$\begin{array}{l}\text { Sagittal \& axial - mandatory } \\
\text { Axial \& coronal - perpendicular and parallel } \\
\text { to cancer axis }\end{array}$} \\
\hline Slice thickness & \multicolumn{2}{|c|}{ 1 3mm, maximum $4 \mathrm{~mm}$} \\
\hline
\end{tabular}

MR machines, and sometimes adding the advanced sequences such as DWI and DCE MRI.

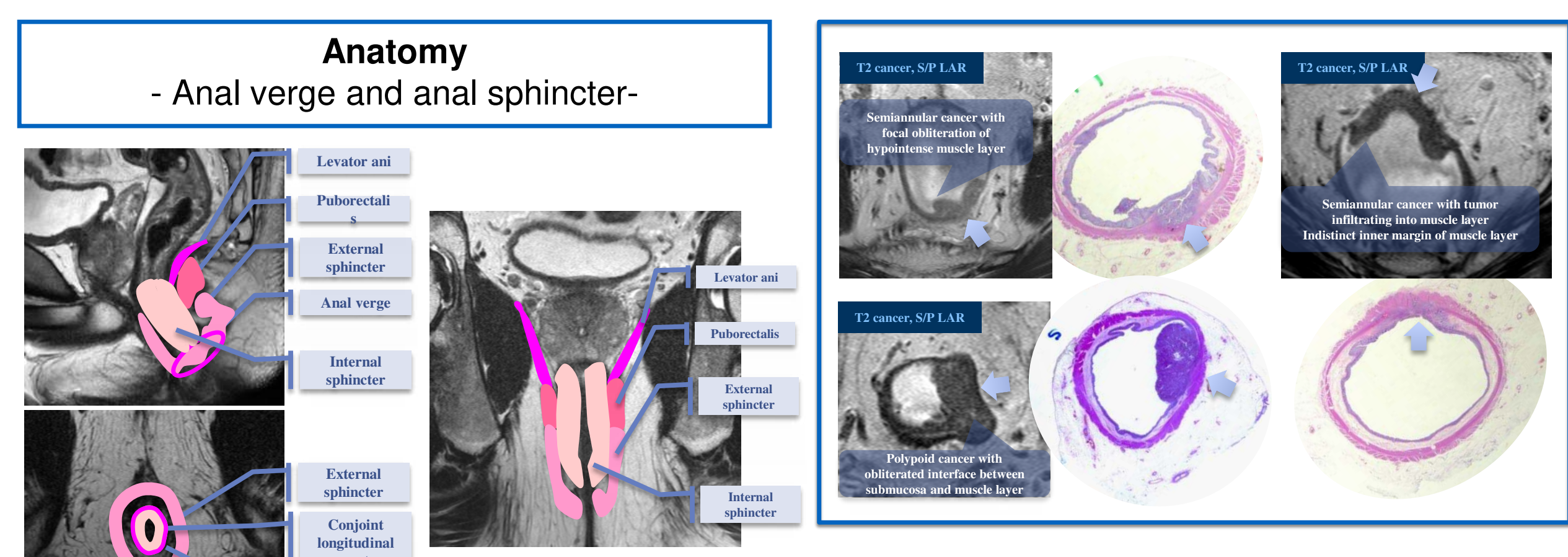
of tumor invasion outside the muscularis propria. The depth of

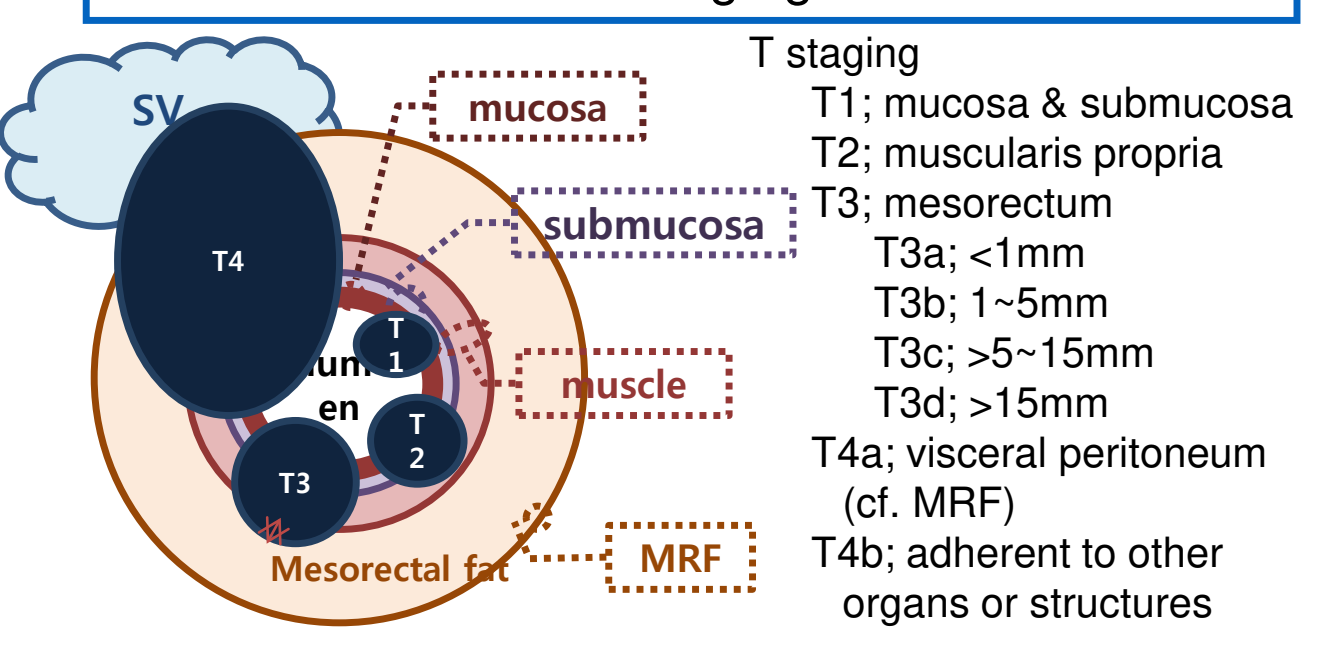

Differentiation of T1 and T2 cancer is not reliable by using MRI. Endorectal ultrasound (US) is still recommended in the evaluation of early rectal cancer. However, high-resolution
MRI can be used in differentiation of T1 and T2 cancer by MRI can be used in differentiation of $\mathrm{T} 1$ and $\mathrm{T} 2$ cancer by
assessing the preservation of interface between hyperintense submucosal layer and hypointense muscularis propria. When this interface is obliterated by intermediate tumor signal intensity, it can be deep submucosal invasion (T1 SM3) or superficial muscle ivasion (T2) which require the sungical intervention. Endoscopir

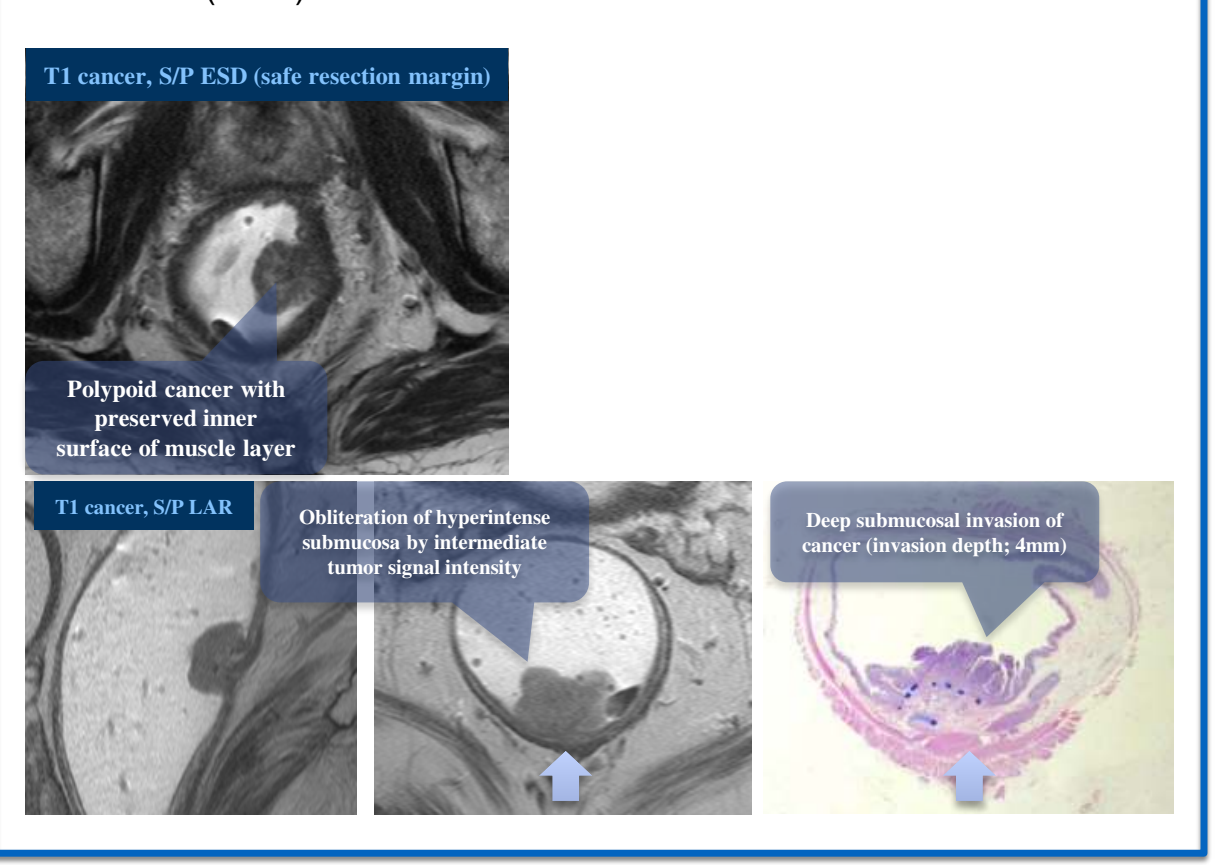

T3 cancer is classified into T3a T3d according to the depth invasion is closely related with survival rate independent of nodal staging. The depth of invasion outside the muscularis propria tumor invasion should be measured from the imaginary muscle tumor invasion
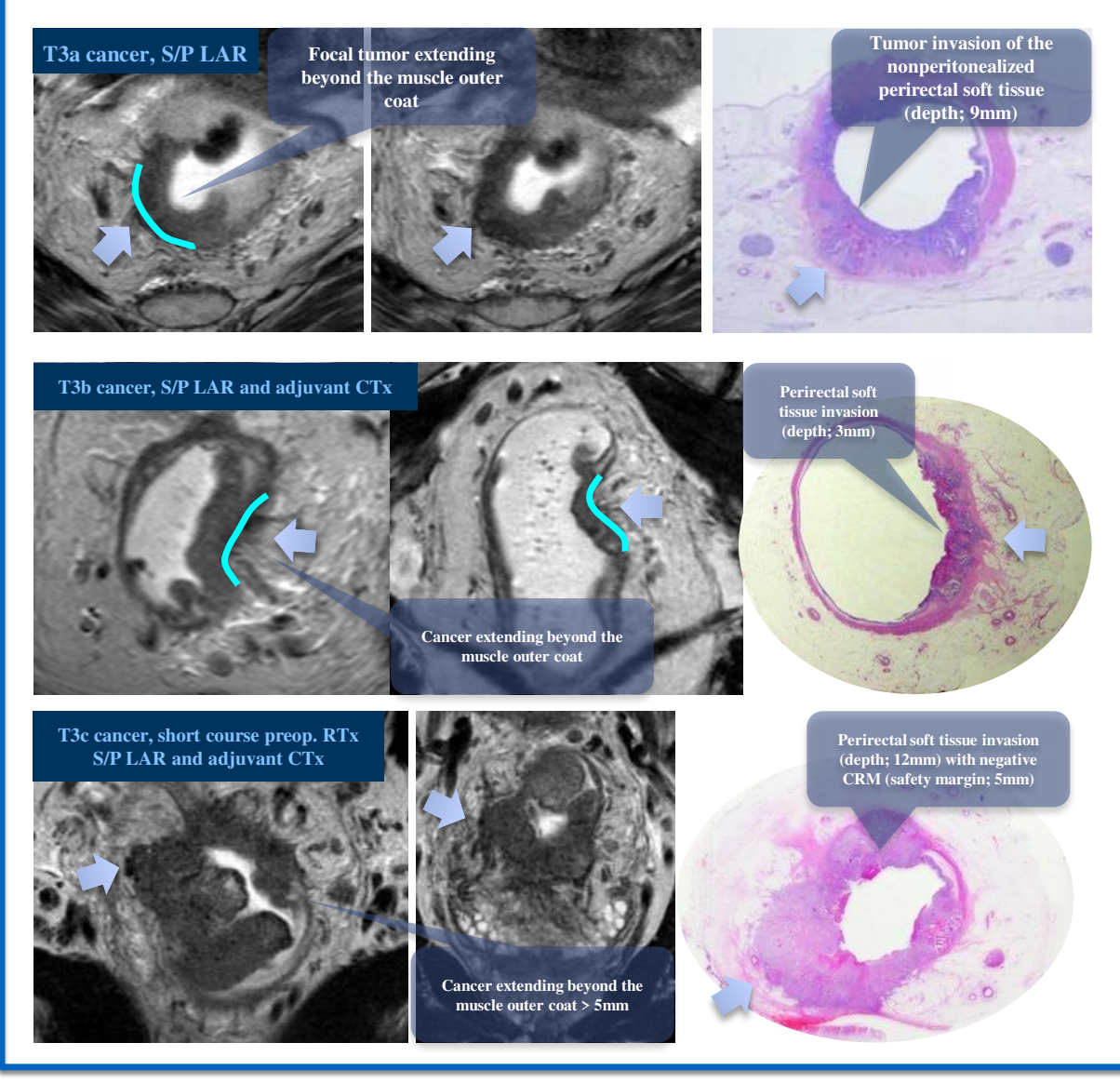

Extramural venous invasion (EMVI)

EMVI is histopathologically defined as the extension of rectal tumor into the veins beyond the muscularis propria. EMVI is frequently demonstrated in rectal MRI. However, it is notoriously under-reported in pathologic examination. It should be detected in at least $25 \%$ of resections for rectal cancer according to Royal college of Pathologist in UK. It is an important clinical predictor of visceral metastasis, relapse, and reduced survival in rectal cancer. In stage II tumors, positive EMVI in preoperative MRI may prompt oncologists to offer adjuvant chemotherapy. Neoadjuvant therapy in positive EMVI can improve disease free survival.

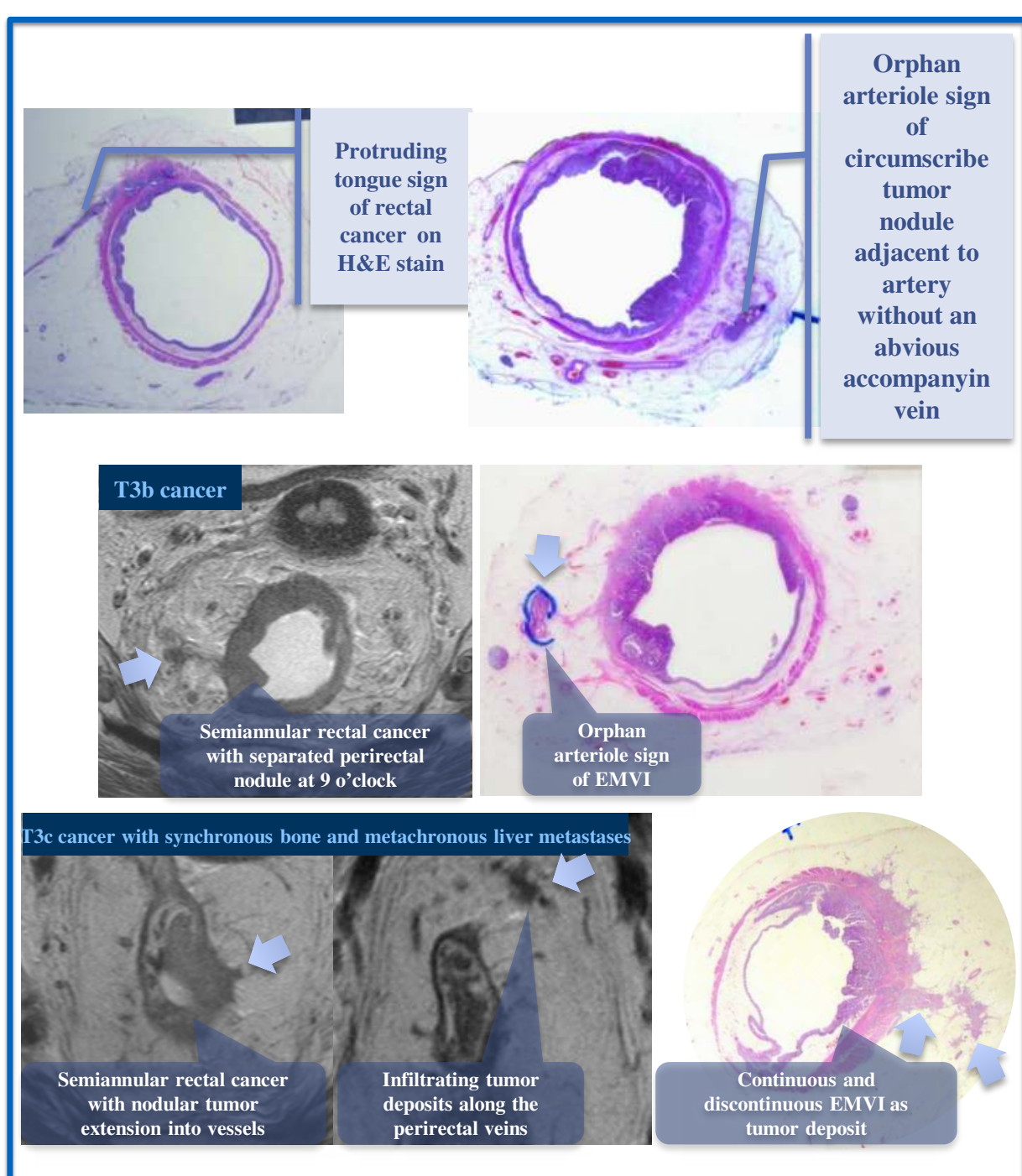

Advanced cancer invading CRM, adjacent organs, or visceral peritoneum requires neoadjuvant $\mathrm{CRT}$ and reassessment of
the treatment response and staging. High-resolution MR has a high negative predictive value for the prediction of tumor invasion into the
adjacent structures such as prostate, seminal vesicle, uterus, urinary bladder, or sacrum.

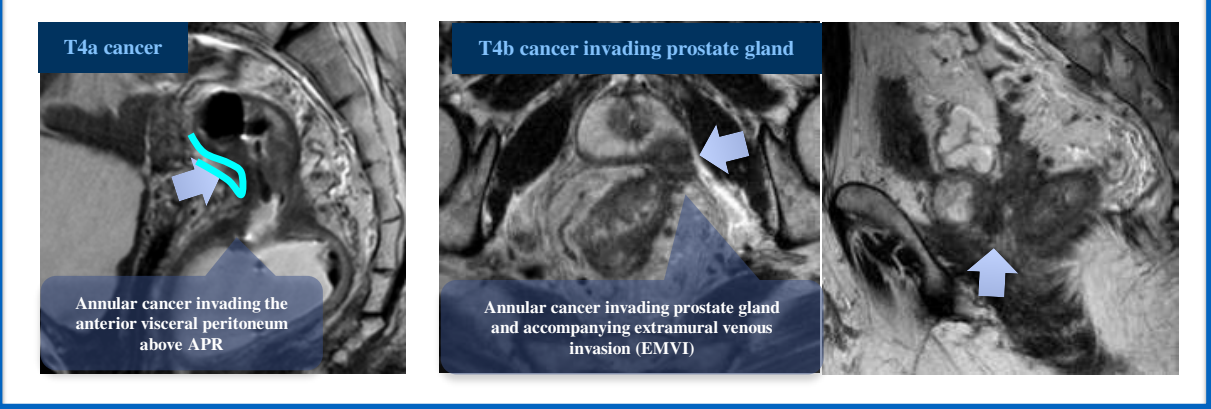

On T2WI, EMVI is suggested when vessels close to the tumor are obviously irregular or expanded by tumor signal EMVI). Therefore, the presence of EMVI should be assessed regardless of $\mathrm{T}$ or $\mathrm{N}$ staging intensity. EMVI would be continued from primary tumor or 